\title{
EUROPEAN SOCIETIES OF CARDIOLOGY
}

\author{
EuROPEAN SOCIETY OF CARDIOLOGY \\ Honorary Presidents: Sir John Parkinson, London \\ Gustave Nylin, Stockholm \\ Evan Bedford, London \\ President: $\quad$ J. Lenegre, Paris \\ Vice-Presidents: $\quad$ L. Condorelli, Rome \\ P. Lukl, Olomouc \\ Secretary: $\quad$ Francois van Dooren, Bruxelles \\ 80, Rue Mercelis, Bruxelles 5, Belgium \\ Treasurer: $\quad$ I. Mahaim, Lausanne \\ Council: $\quad$ R. Cordeiro, Lisbon; K. Shirley Smith, London \\ R. Thauer, Bad-Nauheim; E. J. Warburg, Copenhagen
}

At the request of the Council of the European Society of Cardiology, we are publishing the names of the officials and addresses of the Constituent Societies.

Allemagne

President: Pr. Dr. K. Wezler

Secretary: Pr. Dr. R. Thauer; William G. Kerckhoff Institut, Der Max Planck Geselschaft, Bad Nauheim.

BRITAIN President: Dr. D. Evan Bedford

Chairman: Dr. William Evans

Secretary: Dr. J. P. Shillingford; Postgraduate Medical School, Ducane Road, London, W.12.

Belgique $\quad$ Address: 43, rue des Champs Elysées, Bruxelles 5.

President: Pr. Jean Lequime

Secretary: Dr. Henri Denolin; 178, avenue Winston Churchill, Bruxelles 18.

Meetings: Les deuxièmes Dimanches de Janvier, Mars, Mai, Septembre et Novembre

DANEMARK President:

Secretary: Dr. Henning Gotzche; Cardiac Clinic, University Hospital, Arhus

ESPAGNE

FINLANDE

France

President: Pr. Jean Lenegre

Secretary: Pr. M. Mouquin; Hôspital Broussais la Charite, 96 rue Didot, Paris VIIè. Réunions: Les troisièmes Dimanches de Janvier, Mars, Mai, Octobre, et Décembre

GreECE Address: 14 rue Marni, Athens

President: Pr. G. Michailides

Secretary: Dr. A. Samaras; 14 rue Patr. Ioakeim, Athens 
HollaNde

HONGRIE

IRLANDE

ITALIE

NORVEGE

PolOGNe

Portugal

ROUMANIE

SUEDE

SuISSE

TChecoslovaquie: Adress: Sokolska 31, Praha 2

President: Dr. Pavel Lukl

Secretary: Dr. Arnost Fronek; Budejovicka 800 Praha, Krc.

Meetings: Tous les derniers vendredis du mois

Address: Baross U. 28, Budapest VIII

Presidents: G. Gottsegen, J. Kudasz, I. Kunos, and I. Zarday

Secretary: K. Ghyczy

Meetings: each month

President: Dr. B. Mayne

Secretary: Dr. Sean Blake; Mater Misericordiae Hospital, Dublin

Meetings: Spring, summer, autumn, and winter.

President: Pr. Luigi Condorelli

Secretary: Dr. Vittorio Puddu; 80, via Savoia, Rome

President: Dr. Lar Werkö

Secretary: Dr. Harold Eliasch; Serafimerlasarettet, Stockholm

Meetings: April, September, October, and December

President: Dr. B. Steinmann

Secretary: Dr. P. D. W. Schweizer; Steinenring 3, Bâle

TURQUIE

YougosLAVIE 